\title{
Estudo dos efeitos da acetilação em fibras de sisal
}

\author{
Fernanda F. M. Lopes ${ }^{1}$, G ilmar T. de Araújo일 José W. B. do N ascimento ${ }^{3}$, Tatiana S. Gadelha ${ }^{4}$ \& Valneide R. da Silva ${ }^{5}$
}

\begin{abstract}
RESUMO
0 emprego de fibras vegetais na confecção de compósitos tem grande viabilidade, no que diz respeito ao uso de materiais oriundos de fontes renováveis, à biodegradabilidade e aos benefícios socioeconômicos gerados na produção de matériaprima vegetal. As fibras de sisal são altamente higroscópicas e esta característica se apresenta como um dos principais problemas na produção de compósitos induzindo a variações dimensionais sob a influência da umidade, deposição dos produtos da matriz em seus poros e a degradação. 0 s tratamentos de acetilação nas fibras de sisal foram aplicados em diferentes temperaturas e tempos reacionais, e a eficiência desses tratamentos, considerando-se a redução da hidrofilicidade e a manutenção do desempenho mecânico das fibras, foi avaliada pela capacidade de absorção de água por imersão, ensaios de resistência mecânica e por espectroscopia de infravermel ho. Fibras acetiladas apresentaram reduções de peso por absorção de até $50 \%$ quando comparadas com as não tratadas. 0 s tratamentos por $3 \mathrm{~h}$ apresentaram as maiores perdas na resistência mecânica e a $120^{\circ} \mathrm{C}$ por $1 \mathrm{~h}$ indicaram as melhores características físico-mecânicas, além de incremento satisfatório de grupos apolares com o tratamento.
\end{abstract}

Palavras-chave: Agave sisalana, tratamento de fibras, hidrofilicidade

\section{Study of the effects of acetylation treatments on sisal fiber}

\begin{abstract}
The use of vegetable fibers in composites is highly viable regarding about the use of materials from renewable sources, the biodegradability and the socioeconomic advantages in the production of raw vegetable. The sisal fibers are highly hygroscopic and this is a main problem in the production of composites, inducing dimensional changes under moisture influence, deposition of the matrix products and degradation. The treatment of the acetylation was applied at different temperatures and reaction times, and the efficiency of treatments, considering the reduction of the hydrophilicity and maintenance of the mechanical properties, was evaluated by water sorption, mechanical properties and the infrared spectroscopy. With acetylation the fibers had weight reductions for water sorption up to $50 \%$ compared to untreated. The treatments for $3 \mathrm{~h}$ decreased the mechanical properties while the $120^{\circ} \mathrm{C}$ for $1 \mathrm{~h}$ showed better physico-mechanical properties and increase of apolar groups.
\end{abstract}

Key w ords: Agave sisalana, treatment of fibers, hydrophilicity

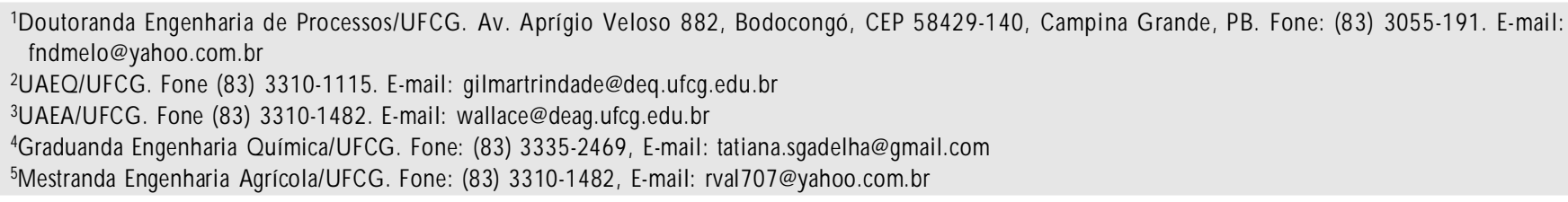




\section{INTRODUÇÃO}

O uso de fibras vegetais na produção de compósitos temse destacado no que se refere a materiais alternativos aplicados para construção de pequenas estruturas. As vantagens nesta utilização não se limitam ao setor econômico mas abrangem, também, outras vertentes importantes, como a aplicação de materiais ecologicamente seguros e que possam trazer benefícios sociais. Nos países em desenvolvimento, como o Brasil, o emprego de fibras de culturas como sisal, cana-deaçúcar, juta e curauá, na indústria, pode levar a uma melhoria de vida das comunidades produtoras que, em sua maioria têm, nessas culturas, uma forma de sobrevivência.

A fibra de sisal é considerada um polímero natural constituído basicamente por celulose, hemicelulose e lignina. Segundo Winandy \& Rowell (1984) os referidos constituintes contribuem diferentemente entre si para a resistência mecânica. A celulose é primariamente responsável pela resistência das fibras, por seu alto grau de polimerização e orientação linear. A hemicelulose atua como matriz para a celulose e aumenta a densidade de empacotamento da parede da célula. A lignina não somente mantém as fibras juntas, mas, também, atua como agente enrijecedor para as moléculas de celulose dentro da parede da fibra. O conhecimento da estrutura interna das fibras ligno-celulósicas e da sua composição química é importante para compreensão de sua influência nas características do compósito e também na escolha dos seus tratamentos químicos e físicos.

De acordo com Gatto et al. (2003), a aplicação de fibras vegetais como reforço permite a obtenção de produtos compósitos que, além de apresentarem menor massa específica aparente e maior porosidade apresentam, ainda, valores satisfatórios de resistência à tração e ao impacto, maior controle de fissuração, além de comportamento dúctil na ruptura. $\mathrm{O}$ compósito, ao invés de romper-se subitamente após o início da fissuração da matriz, apresenta uma deformação plástica considerável que o torna um material adequado para construção (Sarmiento \& Freire, 1997).

Para Silva (2002), a incompatibilidade entre fibra e matriz pode ser classificada de duas formas: a incompatibilidade química, que pode enfraquecer a fibra, e a incompatibilidade física, que pode causar perda de aderência. Savastano Júnior \& Pimentel (2000), afirmaram que a incompatibilidade física pode ser representada pelas variações dimensionais das fibras vegetais sob a influência da umidade e que, ao longo do envelhecimento, um compósito pode apresentar problemas de degradação das fibras e da ligação fibra-matriz.

Ao estudarem diferentes métodos de modificação superficial das fibras de sisal, Rong et al. (2001), concluíram que as fibras podem ser modificadas efetivamente por substâncias químicas e tratamentos físicos. Métodos químicos normalmente provocam uma superfície ativa introduzindo algum grupo reativo e proporcionam, às fibras, maior extensibilidade pela remoção parcial da lignina e hemicelulose. Em contraste, o tratamento térmico pode resultar em maior dureza da fibra devido ao aumento da cristalinidade da celulose. No tratamento de acetilação a eficiência está na modificação química das fibras com anidrido acético, cuja principal função é a substi- tuição das hidroxilas presentes nas paredes das células pelo grupo acetila, condicionando-as um caráter apolar característico deste grupo. Quanto ao ácido acético, sua maior contribuição está diretamente ligada ao intumescimento da fibra.

Visando à potencialidade de emprego e ao problema da degradabilidade das fibras vegetais na confecção de compósitos objetivou-se, com este trabalho, determinar a influência do tratamento químico de acetilação em diferentes níveis, na redução da hidrofilicidade e no desempenho mecânico das fibras de sisal.

\section{MATERIAL E MÉTODOS}

O experimento foi conduzido no Laboratório de Química de Biomassa - Engenharia Química - UFCG, Campina Grande, PB. As fibras de sisal (Agave sisalana) utilizadas foram produzidas no campo experimental da Embrapa Algodão, no município de Pocinhos, PB. Um lote de fibras beneficiadas foi previamente separado para realização dos ensaios. Antes da realização dos tratamentos as fibras de sisal foram lavadas em água destilada, a temperatura ambiente, secadas em estufa a $60{ }^{\circ} \mathrm{C}$, por $24 \mathrm{~h}$, para retirada de qualquer material residual do beneficiamento presente em sua superfície.

\section{Acetilação}

Anterior ao tratamento de acetilação as fibras foram embebidas em uma solução aquosa de $\mathrm{NaOH}$ a $1 \%$ por $1 \mathrm{~h}$; em seguida, foram repetidamente lavadas até que apresentassem pH neutro na solução de lavagem final e secadas em estufa a $60{ }^{\circ} \mathrm{C}$, durante $24 \mathrm{~h}$. Para a realização dos tratamentos de acetilação a metodologia adotada foi adaptada de D'Almeida et al. (2005), onde as amostras de fibras foram acondicionadas em um reator de condensação, sob agitação e em banho termostático com solução de anidrido acético e ácido acético, na proporção de 1,5:1,0 em massa. A cada $200 \mathrm{~mL}$ de solução foram adicionadas 5 gotas de ácido sulfúrico, como catalisador da mistura . Os tempos reacionais (contato entre fibras e solução) foram de 1 e $3 \mathrm{~h}$, a temperaturas de 100 e $120^{\circ} \mathrm{C}$. Decorrido o tempo de reação as amostras foram lavadas inicialmente com água corrente e, finalmente, com destilada, onde permaneceram por $1 \mathrm{~h}$, apresentando $\mathrm{pH}$ entre 5-6 na solução de lavagem final; em seguida, foram secadas em estufa a $60{ }^{\circ} \mathrm{C}$, durante $24 \mathrm{~h}$, e armazenadas em dessecador com sílica gel até o momento dos ensaios.

\section{Ensaios}

$\mathrm{Na}$ determinação da absorção d'água as fibras não tratadas e acetiladas previamente secadas, foram pesadas (cerca de $1 \mathrm{~g}$ ) e imersas em recipientes com água destilada, em seguida, as fibras foram retiradas d'água, secadas superficialmente com papel absorvente e pesadas em balança semianalitíca após 0,5, 2 e 24 h do início do teste. Os resultados foram determinados de acordo com a Eq. 1.

$$
\mathrm{P}(\%)=\left[\left(\mathrm{P}_{1}-\mathrm{P}_{0}\right) / \mathrm{P}_{0}\right]^{*} 100
$$


em que: $\quad P_{0}$ - peso seco inicial, $g$

$\mathrm{P}_{1}$ - peso após imersão em água, $\mathrm{g}$

$\mathrm{Na}$ avaliação do comportamento mecânico das fibras pela resistência a tração utilizou-se uma máquina de ensaios universal, com velocidade de $1 \mathrm{~mm} \mathrm{~min}^{-1}$. Para que as garras da máquina pudessem segurar as fibras, corpos-de-prova foram montados com o auxílio de papel tipo cartolina, nas dimensões de 15 x 2,0 cm. As fibras foram coladas a esses corposde-prova deixando-se um comprimento útil de ensaio de 10 $\mathrm{cm}$. Utilizaram-se 12 corpos-de-prova para cada tratamento. $\mathrm{Na}$ confecção dos resultados foram contabilizados apenas os 8 melhores ensaios, com base no ajuste dos dados experimentais aos calculados. O diâmetro médio das fibras utilizado nos ensaios foi determinado com base na leitura do diâmetro de 30 fibras, obtidos por projeção ampliada e com leitura direta por um projetor de perfil vertical.

Para determinação da presença e intensidade de grupos acetila nas fibras de sisal em decorrência do tratamento de acetilação, utilizou-se a espectroscopia na região do infravermelho avaliando-se comprimentos de onda entre 500 e $4000 \mathrm{~cm}^{-}$ ${ }^{1}$. Para o ensaio, as fibras foram secadas em estufa a $80{ }^{\circ} \mathrm{C}$, por $24 \mathrm{~h}$, de forma que perdessem a maior parte do conteúdo de água livre sobre a superfície sem modificar a sua composição química pela exposição ao calor. Em seguida, as fibras foram picadas, maceradas e prensadas em pastilhas de $\mathrm{NaCl}$. As mudanças morfológicas na superfície das fibras foram realizadas com base nas imagens obtidas por microscopia eletrônica de varredura (MEV), realizadas utilizando-se elétrons secundários e voltagem de aceleração de feixe elétrons entre 15-20 kv.

\section{RESULTADOS E DISCUSSÃO}

\section{Absorção d'água}

Um dos critérios de avaliação e também objetivo deste trabalho, é a redução da absorção d'água pelas fibras de sisal com o tratamento de acetilação devido ao caráter apolar de que este tem capacidade de promovê-la, em consequência das substituições de grupos reativos em suas paredes celulares. Uma inferência do grau de acetilação das fibras pode ser obtida nos testes de absorção d'água por imersão, ao se comparar o comportamento das fibras não tratadas e tratadas por acetilação. Pelos dados da Tabela 1 observa-se que as fibras de sisal não tratadas apresentaram um ganho de peso por absorção d'água de até $86 \%$ em 24 h de imersão e que a comparação dos resultados obtidos entre fibras acetiladas e não tratadas mostrou que os tratamentos de acetilação
Tabela 1. Absorção d'água das fibras de sisal não tratadas e acetiladas

\begin{tabular}{cccc}
\hline & \multicolumn{3}{c}{ Absorção d'água (\%) } \\
\cline { 2 - 4 } Tratamento & \multicolumn{3}{c}{ Tempo de imersão (h) } \\
\cline { 2 - 4 } & $\mathbf{0 , 5}$ & $\mathbf{2}$ & $\mathbf{2 4}$ \\
$\mathrm{N} / \mathrm{T}$ & 77 & 81 & 86 \\
$\mathrm{~A} 1$ & 42 & 44 & 47 \\
$\mathrm{~A} 2$ & 32 & 34 & 36 \\
$\mathrm{~A} 3$ & 30 & 34 & 38 \\
$\mathrm{~A} 4$ & 31 & 33 & 37 \\
\hline
\end{tabular}

N/T - não tratada; Acetilada: $\mathrm{A} 1$ - a $100{ }^{\circ} \mathrm{C}$ por $1 \mathrm{~h} ; \mathrm{A} 2$ - a $100{ }^{\circ} \mathrm{C}$ por

$3 \mathrm{~h}$; A3 - a $120{ }^{\circ} \mathrm{C}$ por $1 \mathrm{~h} ; \mathrm{A} 4-$ a $120{ }^{\circ} \mathrm{C}$ por $3 \mathrm{~h}$

apresentaram eficiência na redução da hidrofilicidade. As fibras acetiladas tiveram um ganho de peso por absorção de 36 a $47 \%$ em 24 h, reduções de 50 e $39 \%$ quando comparadas com as não tratadas. Considerações importantes também podem ser feitas com relação ao aumento da temperatura de reação que, neste caso, apresentou redução média de $10 \%$ em até 24 h, entre tratamentos com $1 \mathrm{~h}$ de reação, apenas pelo aumento da temperatura em $\pm 10^{\circ} \mathrm{C}$. Os tratamentos com $3 \mathrm{~h}$ de reação mostraram comportamento similar, independente da temperatura utilizada. As fibras de sisal acetiladas a $100{ }^{\circ} \mathrm{C}$, por $1 \mathrm{~h}$, indicaram maior capacidade de absorção que nos demais tratamentos; este comportamento pode estar associado a uma reação de acetilação parcial, em que a formação e a deposição do acetato de celulose sobre a superfície da fibra foram insuficientes para causar efeito impermeabilizante na presença de água. Os tratamentos com temperatura de $120^{\circ} \mathrm{C}$ e/ou $3 \mathrm{~h}$ de reação apresentaram absorções similares em todos os tempos analisados, cujos resultados demonstram que a variação desses critérios nem sempre garante eficiência nos tratamentos de acetilação das fibras podendo gerar degradações que impossibilitem sua utilização.

\section{Propriedades mecânicas}

A análise das propriedades mecânicas das fibras de sisal pode contribuir para a detecção de possíveis degradações relacionadas aos tratamentos a que as mesmas são submetidas. A perda da capacidade mecânica gera a impossibilidade de utilização das fibras vegetais, como reforço na confecção de compósitos. Dos tratamentos estudados, apenas a acetilação a $100{ }^{\circ} \mathrm{C}$ por $1 \mathrm{~h}$ apresentou resultados próximos daqueles observados nas fibras não tratadas, sem variações médias consideráveis das propriedades estudadas (Tabela 2). Fibras acetiladas a $120^{\circ} \mathrm{C}$, por $1 \mathrm{~h}$, apresentaram perdas de até $29 \%$ em sua resistência mecânica quando comparadas com as acetiladas a $100{ }^{\circ} \mathrm{C}$ com mesmo tempo reacional; indepen-

Tabela 2. Propriedades mecânicas das fibras de sisal não tratadas e acetiladas

\begin{tabular}{|c|c|c|c|c|c|c|c|c|}
\hline \multirow{2}{*}{ Tratamento } & \multicolumn{2}{|c|}{ Força (N) } & \multicolumn{2}{|c|}{ Alongamento (mm) } & \multicolumn{2}{|c|}{ Tensão (MPa) } & \multicolumn{2}{|c|}{ Módulo de Elasticidade (GPa) } \\
\hline & Min-Máx & Média (Sd) & Min-Máx & Média (Sd) & Min-Máx & Média (Sd) & Min-Máx & Média (Sd) \\
\hline $\mathrm{N} / \mathrm{T}$ & $4,0-15,0$ & $8,6(3,8)$ & $1,0-3,5$ & $2,1(0,8)$ & $101,9-386,3$ & $215,6(95,5)$ & $5,4-16,7$ & $11,5(3,4)$ \\
\hline $\mathrm{A} 1$ & $6,5-11,5$ & $8,6(1,9)$ & $1,4-2,4$ & $1,9(0,3)$ & $163,0-290,0$ & $216,4(48,2)$ & $6,1-12,1$ & $10,1(1,9)$ \\
\hline $\mathrm{A} 2$ & $2,7-8,0$ & $4,7(1,9)$ & $1,1-2,3$ & $1,5(0,4)$ & $68,0-203,0$ & $118,1(48,7)$ & $4,7-7,8$ & $6,7(1,0)$ \\
\hline A3 & $5,1-7,9$ & $6,1(0,8)$ & $1,7-2,1$ & $1,9(0,2)$ & $129,0-199,9$ & $152,7(21,2)$ & $5,9-10,4$ & $7,5(1,3)$ \\
\hline A4 & $1,79-7,8$ & $4,2(1,8)$ & $1,2-2,1$ & $1,4(0,5)$ & $45,1-196,3$ & $106,5(45,0)$ & $3,9-14,8$ & $7,7(3,1)$ \\
\hline
\end{tabular}

N/T - não tratada; Acetilada: A1 - a $100{ }^{\circ} \mathrm{C}$ por $1 \mathrm{~h} ; \mathrm{A} 2$ - a $100{ }^{\circ} \mathrm{C}$ por $3 \mathrm{~h} ; \mathrm{A} 3$ - a $120{ }^{\circ} \mathrm{C}$ por $1 \mathrm{~h} ; \mathrm{A} 4-$ a $120{ }^{\circ} \mathrm{C}$ por $3 \mathrm{~h}$ 
dente da temperatura, as fibras acetiladas por $3 \mathrm{~h}$ apresentaram as maiores perdas na resistência mecânica e ainda comportamento similar, com reduções médias próximas a 50\% nos valores de força e tensão, indicando severa degradação das fibras. Hill et al. (1998), observaram que a resistência a tração das fibras acetiladas a $100{ }^{\circ} \mathrm{C}$, obteve um aumento quando comparada com as fibras não tratadas e que, quando acetiladas a $120{ }^{\circ} \mathrm{C}$, houve redução e esta diminuição da resistência a tração pode estar relacionada à degradação das fibras nesta temperatura. Também salientaram que o aumento da resistência a tração observada nas fibras modificadas a mais baixa temperatura está relacionada a uma redução no conteúdo d'água de equilíbrio, como consequência do aumento da hidrofobicidade.

A análise do comportamento das curvas de tensão da Figura 1 também mostrou similaridade entre fibras de sisal não tratadas e acetiladas a $100{ }^{\circ} \mathrm{C}$, por $1 \mathrm{~h}$, e considerável redução na tensão em fibras acetiladas, principalmente quando acetiladas por $3 \mathrm{~h}$. Também se observa que as fibras acetiladas a $120^{\circ} \mathrm{C}$, por $1 \mathrm{~h}$, levaram maior tempo para ruptura e este fato pode estar relacionado com a maior elasticidade promovida pela retirada parcial da lignina das fibras pelo tratamento.

\section{Espectroscopia de infravermelho (FTIR)}

Tem-se, na Figura 1, os espectros de infravermelho (FTIR) das fibras de sisal não tratadas e acetiladas. Ao se observar o comportamento obtido pela fibra não-tratada com relação aos demais tratamentos, comprova-se que houve modificações na composição das fibras. As fibras de sisal natural apresentam alta intensidade de pico a $3300-3500 \mathrm{~cm}^{-1}$, em decorrência da vibração axial, característica das hidroxilas (O$\mathrm{H})$ neste comprimento de onda, e nas fibras acetiladas a presença desta banda também foi observada. A presença desses grupos após o tratamento de acetilação pode estar relacionada a uma reação incompleta e, apesar da esterificação desse grupo não ter sido total, é potencialmente interessante, pois reduz a higroscopicidade das fibras (D'Almeida et al., 2005). A ocorrência desses picos nas fibras acetiladas pode estar relacionada ao grau de acetilação aplicado neste trabalho, preservando a constituição de alguns elementos. Também de acordo com D'Almeida et al. (2005), as variações observadas nas bandas características de grupamento carbonilas, de 1636$1750 \mathrm{~cm}^{-1}$, e de vibração axial das hidroxilas, de $3300-3500 \mathrm{~cm}^{-}$ 1 , permitem uma análise da eficiência do tratamento de acetilação. Ainda foi possível observar um incremento na banda em $1750 \mathrm{~cm}^{-1}$, correspondente à vibração do grupo carbonila $(\mathrm{C}=\mathrm{O})$, gerados a partir da esterificação das hidroxilas presentes nas fibras (Tserki et al., 2005). Ao estudarem a modificação química nas fibras de coco e sisal pela acetilação, Kapulskis et al. (2003), concluíram que o aumento dos grupos carbonílicos indica que a reação tenha ocorrido de fato e que a presença dessas bandas em fibras não tratadas se deve, possivelmente, a uma oxidação natural das mesmas.

Em $1650 \mathrm{~cm}^{-1}$ a intensidade do pico para a fibra acetilada diminui sensivelmente, o aparecimento ou modificação deste pico e pode estar associado ao potencial de absorção de água pela celulose cristalina (Tserki et al., 2005). O estiramento
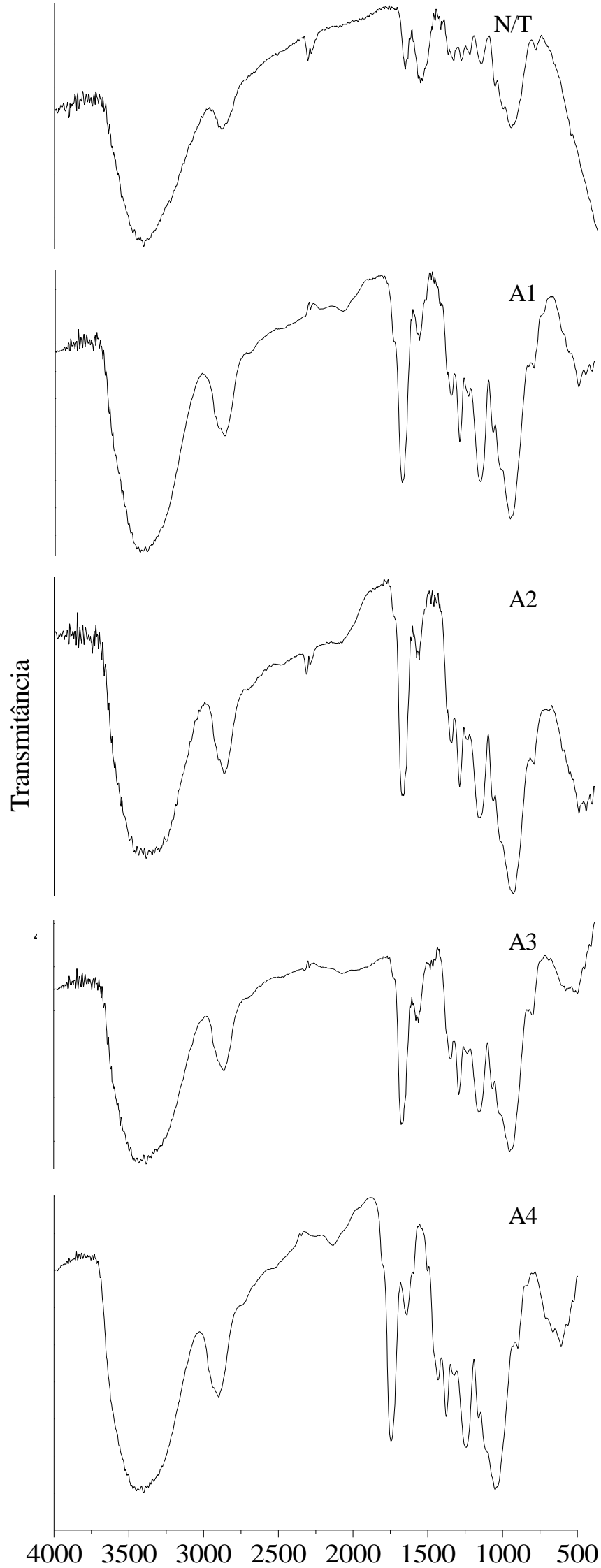

Comprimento de onda $\left(\mathrm{cm}^{-1}\right)$

Figura 1. Espectroscopia de infravermelho das fibras de sisal não tratadas $e$ modificadas por acetilação; $\mathrm{N} / \mathrm{T}$ - não tratada; Acetilada: $\mathrm{A} 1$ - a $100^{\circ} \mathrm{C}$ por $1 \mathrm{~h}$; $\mathrm{A} 2$ - a $100{ }^{\circ} \mathrm{C}$ por $3 \mathrm{~h} ; \mathrm{A} 3-\mathrm{a} 120{ }^{\circ} \mathrm{C}$ por $1 \mathrm{~h} ; \mathrm{A} 4-\mathrm{a} 120{ }^{\circ} \mathrm{C}$ por $3 \mathrm{~h}$ 
ocorrido nas fibras acetiladas a $1380 \mathrm{~cm}^{-1}$ tem correlação com as deformações ocorridas nos acoplamentos C-H e a $1250 \mathrm{~cm}^{-}$ 1 com os acoplamentos C-O. Ainda segundo Tserki et al. (2005), a reação de esterificação também é confirmada pelo

A.

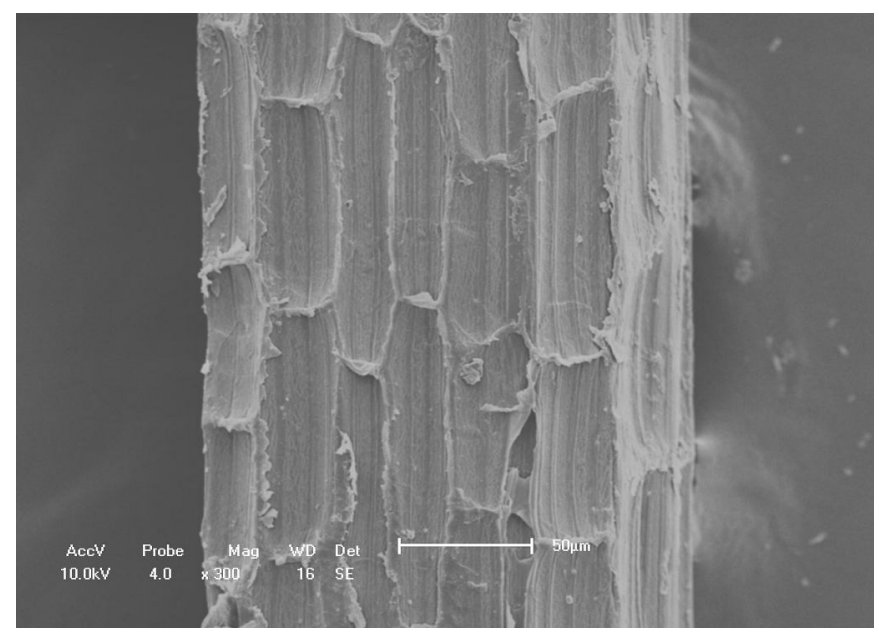

B.

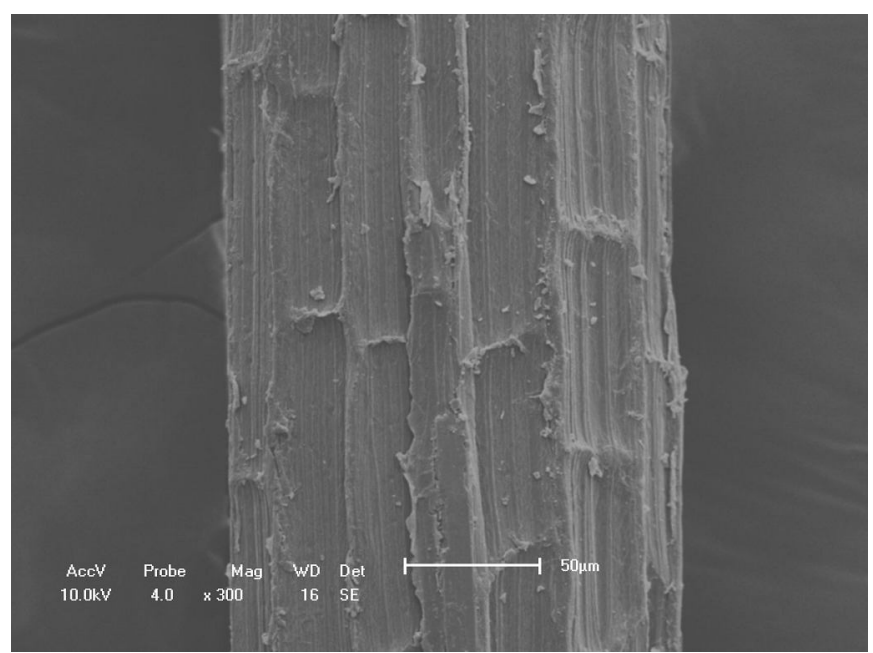

C.

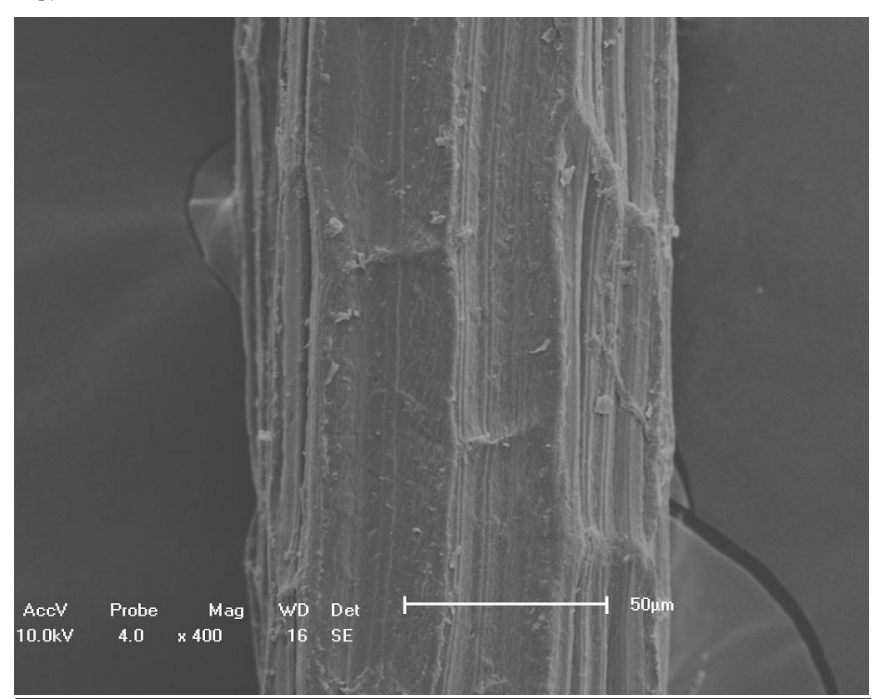

Figura 2. Micrografias da superfície das fibras de sisal sem tratamento (A) e acetiladas por $1 \mathrm{~h}$ a $100^{\circ} \mathrm{C}$ (B) e $120^{\circ} \mathrm{C}$ (C). aparecimento de pico a $1162-1229 \mathrm{~cm}^{-1}$, atribuído ao estiramento C-O do grupo de ésteres carboxílicos.

Lu et al. (2003) observaram, estudando a utilização de fibras de sisal benziladas no reforço de matrizes para confecção de compósitos, que a redução na intensidade dos picos de $\mathrm{C}=\mathrm{O}$ a 1636 e $1250 \mathrm{~cm}^{-1}$, demonstrando que a lignina e a hemicelulose presentes na fibra natural são parcialmente removidas após o tratamento; isto pode ser uma evidência da efetividade e da eficiência dos tratamentos nas fibras. Outra observação pode ser feita a $1037 \mathrm{~cm}^{-1}$, em que este comprimento de onda pode estar associado às deformações nos acoplamentos $\mathrm{C}-\mathrm{H}$ e C-O.

Em estudo sobre os efeitos da acetilação em fibras de empty fruit bunch (EFB) e coco, realizado por Khalil et al. (2001) constatou-se que as mudanças químicas mais significativas ocorridas nas fibras se deram com estiramento na região das carbonilas $\left(1750 \mathrm{~cm}^{-1}\right)$ e nas ligações carbono-hidrogênio (1375 $\left.\mathrm{cm}^{-1}\right)$ e $\left(1238 \mathrm{~cm}^{-1}\right)$, aproximadamente, fato também observado em todas as condições de acetilação estudadas para fibras de sisal neste trabalho e, ainda, que estiramentos em comprimentos entre $2900-3000 \mathrm{~cm}^{-1}$ podem estar relacionados ao estiramento de ligações $\mathrm{C}-\mathrm{H}$ nos anéis aromáticos.

\section{Análise morfológica (MEV)}

A análise morfológica da superfície das fibras obtidas a partir das micrografias pode dar inferências a respeito da eficiência com que os tratamentos são aplicados. As fibras de sisal naturais, Figura 2 A, apresentaram superficialmente células de parênquima bem definidas. Nas fibras acetiladas a 100 ${ }^{\circ} \mathrm{C}$ (Figura $2 \mathrm{~B}$ ), as estruturas das células não são bem definidas como nas fibras não tratadas. Quando acetiladas a 120 ${ }^{\circ} \mathrm{C}$ (Figura $2 \mathrm{C}$ ), as fibras passaram a apresentar uma superfície mais lisa, o que, segundo Bessadok et al. (2009), está associado à eliminação de substâncias que influenciam diretamente na hidrofilicidade como a pectina, a lignina e a cera amorfa presente nas cutículas das fibras.

\section{CONCLUSÕES}

1. O tratamento de acetilação promoveu redução da hidrofilicidade nas fibras de sisal, em todas as condições estudadas.

2. As fibras acetiladas por $3 \mathrm{~h}$ apresentaram reduções mais acentuadas na resistência mecânica, principalmente quanto à força e alongamento máximos suportados até a ruptura, demonstrando enrijecimento na estrutura das fibras.

3. A acetilação a $120^{\circ} \mathrm{C}$ por $1 \mathrm{~h}$ apresentou as melhores características físico-mecânicas dentre os tratamentos estudados com redução considerável da hidrofilicidade, perdas toleráveis nas propriedades mecânicas e incremento satisfatório de grupos apolares com o tratamento.

\section{LITERATURA CITADA}

Bessadok, A.; Langevin, D.; Gouanvé, F.; Chappey, C.; Roudesli, S.; Marais, S. Study of water sorption on modiûed Agave ûbres. Carbohydrate Polymers, v.76, p.74-85, 2009. 
D’Almeida, A. L. F. S.; Calado, V.; Barreto, D. W.; D’Almeida, J. R. M. Acetilação da fibra de bucha (Luffa cylindrica). Polímeros: Ciência e Tecnologia, v.15, n.001, p.59-62, 2005.

Gatto E. G.; Kawabata C. Y.; Savastano Júnior, H. Polpas kraft de Agave sisalana e de Musa cavendishii para reforço de fibrocimento alternativo. Revista Engenharia Agrícola, v.23, n.2, p.211-220, 2003.

Hill C. A. S.; Khalil, H. P. S. A.; Hale, M. D. A study of the potential of acetylation to improve the properties of plant fibres. Industrial Crops and Products, v.8, p.53-63, 1998.

Kapulskis, T. A.; Jesus, R. C.; Mei, L. H. I. Modificação química de fibras de coco e de sisal visando melhorar suas interações interfaciais com matrizes poliméricas biodegradáveis. Congresso Brasileiro de Engenharia Química em Iniciação Científica, 4, 2003, Campinas. Anais... Campinas: UNICAMP, 2003. 6p.

Khalil H. P. S. A.; Ismail H.; Rozman H. D.; Ahmad M. N. The effect of acetylation on interfacial shear strength between plant fibres and various matrices. European Polymer Journal, v.37, p.1037-1045, 2001.

Lu, X.; Zhang, M. Q.; Rong, M. Z.; Shi, G.; Yang, G. C. Self-reinforced melt processable composites of sisal. Composites Science and Technology, v.63, p.177-186, 2003.
Rong, M. Z.; Zhang, M. Q.; Liu, Y.; Yang, G. C.; Zeng, H. M. The effect of fiber treatment on the mechanical properties of unidirectional sisal-reinforced epoxy composites. Composites Science and Technology, v.61, p.1437-1447, 2001.

Sarmiento, C. R.;Freire, W. J. Argamassa de cimento e areia combinada com fibras de bagaço de cana-de-açúcar. Revista Engenharia Agrícola, v.17, n.2, p.1-8, 1997.

Savastano Júnior, H.; Pimentel, L. L. Viabilidade do aproveitamento de resíduos de fibras vegetais para fins de obtenção de material de construção. Revista Brasileira de Engenharia Agrícola e Ambiental, v.4, n.1, p.103-110, 2000.

Silva, A. C. Estudo da durabilidade de compósitos reforçados com fibras de celulose. São Paulo: Escola Politécnica/USP, 2002. 128p. Dissertação Mestrado

Tserki, V.; Zafeiropoulos, N. E.; Simon, F.; Panayiotou, C. A study of the effect of acetylation and propionylation surface treatments on natural fibres. Composites Part A: Applied Science and Manufacturing, v.36, p.1110-1118, 2005.

Winandy, J. E.; Rowel, R. M. The chemistry of wood strength. Advances in the Chemistry Series, v.207, p.311-655, 1984. 